\title{
Relations on the Apostol Type $(p, q)$-Frobenius-Euler Polynomials and Generalizations of the Srivastava-Pintér Addition Theorems
}

\author{
Burak Kurt* \\ Department of Mathematics, Faculty of Educations, University of Akdeniz \\ *Corresponding author: burakkurt@akdeniz.edu.tr
}

Received December 22, 2016; Revised April 19, 2017; Accepted June 14, 2017

\begin{abstract}
In this work, we define and introduce a new kind of the Apostol type Frobenius-Euler polynomials based on the $(p, q)$-calculus and investigate their some properties, recurrence relationships and so on. We give some identities at this polynomial. Moreover, we get $(p, q)$-extension of Carlitz's main result in [1].
\end{abstract}

Keywords: Generating function, Frobenius-Euler polynomials and numbers, (p, q)-calculus, (p, q)-FrobeniusEuler polynomials, Apostol-Bernoulli number and polynomials, generalized q-Bernoulli polynomials, generalized q-Euler polynomials.

Cite This Article: Burak Kurt, "Relations on the Apostol Type $(p, q)$-Frobenius-Euler Polynomials and Generalizations of the Srivastava-Pintér Addition Theorems." Turkish Journal of Analysis and Number Theory, vol. 5, no. 4 (2017): 126-131. doi: 10.12691/tjant-5-4-2.

\section{Introduction, Definitions and Notations}

Throughout this paper, we always make use of the following notation; $\mathbb{N}$ denotes the set of natural numbers, $\mathbb{N}_{0}$ denotes the set of nonnegative integers, $\mathbb{R}$ denotes the set of real numbers and $\mathbb{C}$ denotes the set of complex numbers.

The $(p, q)$-numbers are defined by

$$
[n]_{p, q}=\frac{p^{n}-q^{n}}{p-q}
$$

which is natural generalization of the $q$-number such that

$$
\lim _{p \rightarrow 1}[n]_{p, q}=[n]_{q} \text {. }
$$

Note that $(p, q)$-number is symmetric: that is $[n]_{p, q}=[n]_{q, p}$.

The $(p, q)$-derivative of a function $\mathrm{f}$ is defined by

$$
D_{p, q: x} f(x):=D_{p, q} f(x)=\frac{f(p x)-f(q x)}{(p-q) x},(x \neq 0) .
$$

The $(p, q)$-Gauss Binomial formula is defined by

$$
(x+y)_{p, q}^{n}=\sum_{k=0}^{n}\left[\begin{array}{l}
n \\
k
\end{array}\right]_{p, q} p^{\left(\begin{array}{l}
k \\
2
\end{array}\right) q^{\left(\begin{array}{l}
n-k \\
2
\end{array}\right)} x^{n-k} y^{k}}
$$

where the notations $\left[\begin{array}{l}n \\ k\end{array}\right]_{p, q}((p, q)$-Gauss Binomial coefficients) and $[n]_{p, q} !((p, q)$-factorial) are defined by

$$
\begin{aligned}
& {[n]_{p, q} !=[n]_{p, q}[n-1]_{p, q} \ldots[2]_{p, q}[1]_{p, q},} \\
& {\left[\begin{array}{l}
n \\
k
\end{array}\right]_{p, q}=\frac{[n]_{p, q} !}{[n-k]_{p, q} ![k]_{p, q} !},(n \geq k) .}
\end{aligned}
$$

The $(p, q)$-exponential functions, $e_{p, q}(z)$ and $E_{p, q}(z)$, are defined by

$$
e_{p, q}(x)=\sum_{n=0}^{\infty} \frac{1}{[n]_{p, q} !} p^{\left(\begin{array}{l}
n \\
2
\end{array}\right)} x^{n}
$$

and

$$
E_{p, q}(x)=\sum_{n=0}^{\infty} \frac{1}{[n]_{p, q} !} q^{\left(\begin{array}{l}
n \\
2
\end{array}\right)} x^{n} .
$$

From this form, we easily see that $e_{p, q} E_{p, q}(-z)=1$.

In this work, we introduce Apostol type $(p, q)$-Frobenius-Euler polynomials. We give some new identities for the Apostol type $(p, q)$-Frobenius-Euler polynomials. Also, we prove some explicit expressions.

Definition 1. Let $p, q \in \mathbb{C}, \alpha \in \mathbb{N}$. The (p,q)-Bernoulli numbers $\mathcal{B}_{n, p, q}$ and polynomials $\mathcal{B}_{n, p, q}(x, y)$ are defined by means of the generating functions in [6]:

$$
\sum_{n=0}^{\infty} \mathcal{B}_{n, p, q} \frac{t^{n}}{[n]_{p, q} !}=\left(\frac{t}{e_{p, q}(t)-1}\right),|t|<2 \pi,
$$




$$
\begin{aligned}
& \sum_{n=0}^{\infty} \mathcal{B}_{n, p, q}(x, y) \frac{t^{n}}{[n]_{p, q} !} \\
& =\left(\frac{t}{e_{p, q}(t)-1}\right) e_{p, q}(t x) E_{p, q}(t y),|t|<2 \pi .
\end{aligned}
$$

Definition 2. Let $p, q \in \mathbb{C}, \quad \alpha \in \mathbb{N}$. The $(p, q)$-Euler numbers $\mathcal{E}_{n, p, q}$ and polynomials $\mathcal{E}_{n, p, q}(x, y)$ are defined by means of the generating functions in [6]:

$$
\begin{aligned}
& \sum_{n=0}^{\infty} \mathcal{E}_{n, p, q} \frac{t^{n}}{[n]_{p, q} !}=\left(\frac{[2]_{p, q}}{e_{p, q}(t)+1}\right),|t|<\pi, \\
& \sum_{n=0}^{\infty} \mathcal{E}_{n, p, q}(x, y) \frac{t^{n}}{[n]_{p, q} !} \\
& =\left(\frac{[2]_{p, q}}{e_{p, q}(t)+1}\right) e_{p, q}(t x) E_{p, q}(t y),|t|<\pi .
\end{aligned}
$$

Definition 3. Let $p, q \in \mathbb{C}, \alpha \in \mathbb{N}$. The $(p, q)$-Bernoulli numbers $\mathcal{B}_{n, p, q}^{(\alpha)}$ and polynomials $\mathcal{B}_{n, p, q}^{(\alpha)}(x, y)$ in $x, y$ of order $\alpha$ are defined by means of the generating functions in [6]:

$$
\begin{aligned}
& \sum_{n=0}^{\infty} \mathcal{B}_{n, p, q}^{(\alpha)} \frac{t^{n}}{[n]_{p, q} !}=\left(\frac{t}{e_{p, q}(t)-1}\right)^{\alpha},|t|<2 \pi, \\
& \sum_{n=0}^{\infty} \mathcal{B}_{n, p, q}(x, y) \frac{t^{n}}{[n]_{p, q} !} \\
& =\left(\frac{t}{e_{p, q}(t)-1}\right)^{\alpha} e_{p, q}(t x) E_{p, q}(t y),|t|<2 \pi .
\end{aligned}
$$

Definition 4. Let $p, q \in \mathbb{C}, \quad \alpha \in \mathbb{N}$. The ( $p, q)$-Euler numbers $\mathcal{E}_{n, p, q}^{(\alpha)}$ and polynomials $\mathcal{E}_{n, p, q}^{(\alpha)}(x, y)$ in $x, y$ of order $\alpha$ are defined by means of the generating functions in [6]:

$$
\begin{aligned}
& \sum_{n=0}^{\infty} \mathcal{E}_{n, p, q}^{(\alpha)} \frac{t^{n}}{[n]_{p, q} !}=\left(\frac{[2]_{p, q}}{e_{p, q}(t)+1}\right)^{\alpha},|t|<\pi \\
& \sum_{n=0}^{\infty} \mathcal{E}_{n, p, q}^{(\alpha)}(x, y) \frac{t^{n}}{[n]_{p, q} !} \\
& =\left(\frac{[2]_{p, q}}{e_{p, q}(t)+1}\right)^{\alpha} e_{p, q}(t x) E_{p, q}(t y),|t|<\pi .
\end{aligned}
$$

Classical Frobenius-Euler polynomials $\mathcal{H}_{n}^{(\alpha)}(x ; u)$ of order $\alpha$ is defined by the following relation $[1,7,10,11]$.

$$
\sum_{n=0}^{\infty} \mathcal{H}_{n}^{(\alpha)}(x ; u) \frac{t^{n}}{n !}=\left(\frac{1-u}{e^{t}-u}\right)^{\alpha} e^{x t}
$$

where $\alpha \in \mathbb{N}, u$ algebraic number.
Similarly Frobenius-Euler polynomials $\mathcal{H}_{n}^{(\alpha)}(x ; u ; \lambda)$ of order $\alpha$ is defined by the following relation ([17])

$$
\sum_{n=0}^{\infty} \mathcal{H}_{n}^{(\alpha)}(x ; u ; \lambda) \frac{t^{n}}{n !}=\left(\frac{1-u}{\lambda e^{t}-u}\right)^{\alpha} e^{x t}
$$

Definition 5. The Apostol type q-Frobenius-Euler polynomials $\mathcal{H}_{n, q}^{(\alpha)}(x, y ; u ; \lambda)$ of order $\alpha$ in $x, y$ and Apostol type q-Frobenius-Euler number $\mathcal{H}_{n, q}^{(\alpha)}(0,0 ; u ; \lambda)$ of order $\alpha$, in [9] respectively

$$
\begin{gathered}
\sum_{n=0}^{\infty} \mathcal{H}_{n, q}^{(\alpha)}(x, y ; u ; \lambda) \frac{t^{n}}{n !}=\left(\frac{1-u}{\lambda e_{q}(t)-u}\right)^{\alpha} e_{q}(t x) E_{q}(t y), \\
\sum_{n=0}^{\infty} \mathcal{H}_{n, q}^{(\alpha)}(0,0 ; u ; \lambda) \frac{t^{n}}{n !}=\left(\frac{1-u}{\lambda e_{q}(t)-u}\right)^{\alpha} .
\end{gathered}
$$

Definition 6. Let $p, q \in \mathbb{C}, \quad \alpha \in \mathbb{N}$ and $0<\left|\frac{p}{q}\right|<1$. We define the Apostol type (p, q)-Bernoulli polynomials $\mathcal{B}_{n, p, q}^{(\alpha)}(x, y ; u ; \lambda)$ of order $\alpha$ in $x, y$ and the Apostol type $(p, q)$-Bernoulli numbers $\mathcal{B}_{n, p, q}^{(\alpha)}(0,0 ; u ; \lambda)$ of order $\alpha$ in $x$, $y$ respectively

$$
\begin{gathered}
\sum_{n=0}^{\infty} \mathcal{B}_{n, p, q}^{(\alpha)}(x, y ; u ; \lambda) \frac{t^{n}}{[n]_{p, q} !} \\
=\left(\frac{t}{\lambda e_{p, q}(t)-1}\right)^{\alpha} e_{q}(t x) E_{q}(t y), \\
\sum_{n=0}^{\infty} \mathcal{B}_{n, p, q}^{(\alpha)}(0,0 ; u ; \lambda) \frac{t^{n}}{[n]_{p, q} !}=\left(\frac{t}{\lambda e_{p, q}(t)-1}\right)^{\alpha} .
\end{gathered}
$$

Definition 7. Let $p, q \in \mathbb{C}, \quad \alpha \in \mathbb{N}$ and $0<\left|\frac{p}{q}\right|<1$. We define the Apostol type (p, q)-Euler polynomials $\mathcal{E}_{n, p, q}^{(\alpha)}(x, y ; u ; \lambda)$ of order $\alpha$ in $x, y$ and the Apostol type $(p, q)$-Euler numbers $\mathcal{E}_{n, p, q}^{(\alpha)}(0,0 ; u ; \lambda)$ of order $\alpha$ in $x, y$ respectively

$$
\begin{gathered}
\sum_{n=0}^{\infty} \mathcal{E}_{n, p, q}^{(\alpha)}(x, y ; u ; \lambda) \frac{t^{n}}{[n]_{p, q} !} \\
=\left(\frac{[2]_{p, q}}{\lambda e_{p, q}(t)+1}\right)^{\alpha} e_{q}(t x) E_{q}(t y), \\
\sum_{n=0}^{\infty} \mathcal{E}_{n, p, q}^{(\alpha)}(0,0 ; u ; \lambda) \frac{t^{n}}{[n]_{p, q} !}=\left(\frac{[2]_{p, q}}{\lambda e_{p, q}(t)+1}\right)^{\alpha} .
\end{gathered}
$$

Definition 8. We de.ne Apostol type (p, q)-FrobeniusEuler polynomials $\mathcal{H}_{n, p, q}^{(\alpha)}(x, y ; u ; \lambda)$ of order $\alpha$ in $x, y$ 
and Apostol type (p,q)-Frobenius-Euler numbers $\mathcal{H}_{n, p, q}^{(\alpha)}(0,0 ; u ; \lambda)$ of order $\alpha$, respectively

$$
\begin{gathered}
\sum_{n=0}^{\infty} \mathcal{H}_{n, p, q}^{(\alpha)}(x, y ; u ; \lambda) \frac{t^{n}}{[n]_{p, q} !} \\
=\left(\frac{1-u}{\lambda e_{p, q}(t)-u}\right)^{\alpha} e_{p, q}(t x) E_{p, q}(t y), \\
\sum_{n=0}^{\infty} \mathcal{H}_{n, p, q}^{(\alpha)}(0,0 ; u ; \lambda) \frac{t^{n}}{[n]_{p, q} !}=\left(\frac{1-u}{\lambda e_{p, q}(t)-u}\right)^{\alpha} .
\end{gathered}
$$

Letting $\lim _{p \rightarrow 1}$ in (1.7), we have

$$
\begin{gathered}
\lim _{p \rightarrow 1} \sum_{n=0}^{\infty} \mathcal{H}_{n, p, q}^{(\alpha)}(x, y ; u ; \lambda) \frac{t^{n}}{[n]_{p, q} !} \\
=\lim _{p \rightarrow 1}\left(\frac{1-u}{\lambda e_{p, q}(t)-u}\right)^{\alpha} e_{p, q}(t x) E_{p, q}(t y) \\
\sum_{n=0}^{\infty} \mathcal{H}_{n, q}^{(\alpha)}(x, y ; u ; \lambda) \frac{t^{n}}{[n]_{q} !}=\left(\frac{1-u}{\lambda e_{q}(t)-u}\right)^{\alpha} e_{q}(t x) E_{q}(t y)
\end{gathered}
$$

[9].

Putting $\lambda=1$ and $u=-1$ in (1.7), we have

$$
\mathcal{H}_{n, p, q}^{(\alpha)}(x, y ;-1 ; 1)=\mathcal{E}_{n, p, q}^{(\alpha)}(x, y),
$$

where $\mathcal{E}_{n, p, q}^{(\alpha)}(x, y)$ is $(p, q)$-Euler polynomials of order $\alpha$.

Using $\lim _{p \rightarrow 1}$ in last equation, we have

$$
\mathcal{H}_{n, q}^{(\alpha)}(x, y ;-1 ; 1)=\mathcal{E}_{n, q}^{(\alpha)}(x, y),
$$

where $\mathcal{E}_{n, q}^{(\alpha)}(x, y)$ is $q$-Euler polynomials of order $\alpha$.

Letting $\lim$ in last equation, we have

$$
\mathcal{H}_{n}^{(\alpha)}(x, y ;-1 ; 1)=\mathcal{E}_{n}^{(\alpha)}(x, y),
$$

where $\mathcal{E}_{n}^{(\alpha)}(x, y)$ is Hermite based Euler polynomials of order $\alpha$.

\section{Some Basic Properties for the Apostol Type $q$-Frobenius-Euler Polynomials}

Proposition 1. Apostol type Frobenius-Euler polynomials satisfy the following relations

$$
\begin{aligned}
& \mathcal{H}_{n, p, q}^{(\alpha+\beta)}(x, y ; u ; \lambda) \\
& =\sum_{k=0}^{n}\left[\begin{array}{l}
n \\
k
\end{array}\right]_{p, q} \mathcal{H}_{k, p, q}^{(\alpha)}(x, y ; u ; \lambda) \mathcal{H}_{n-k, p, q}^{(\beta)}(0,0 ; u ; \lambda),
\end{aligned}
$$

$$
\begin{aligned}
& \lambda \sum_{k=0}^{n}\left[\begin{array}{l}
n \\
k
\end{array}\right]_{p, q} \mathcal{H}_{k, p, q}(x, y ; u ; \lambda)-\mathcal{H}_{n, p, q}(x, y ; u ; \lambda) \\
& =(1-u)(x+y)_{p, q}^{n}, \\
& \mathcal{H}_{n, p, q}^{(\alpha-\beta)}(x, y ; u ; \lambda) \\
& =\sum_{k=0}^{n}\left[\begin{array}{l}
n \\
k
\end{array}\right]_{p, q} \mathcal{H}_{k, p, q}^{(\alpha)}(x, 0 ; u ; \lambda) \mathcal{H}_{n-k, p, q}^{(-\beta)}(0, y ; u ; \lambda) .
\end{aligned}
$$

Theorem 1. For $n \in \mathbb{N}_{0}$ and $x, y, a \in \mathbb{C}$, the following relationships hold true:

$$
\begin{aligned}
& \mathcal{H}_{n, p, q}^{(\alpha)}(x+a, y ; u ; \lambda) \\
& =\sum_{k=0}^{n}\left[\begin{array}{l}
n \\
k
\end{array}\right]_{p, q} \mathcal{H}_{n-k, p, q}^{(\alpha)}(0, y ; u ; \lambda) p^{\left(\begin{array}{l}
k \\
2
\end{array}\right)} \sum_{s=0}^{k}\left(\begin{array}{l}
k \\
s
\end{array}\right) x^{s} a^{k-s}, \\
& \mathcal{H}_{n, p, q}^{(\alpha)}(x, y+a ; u ; \lambda) \\
& =\sum_{k=0}^{n}\left[\begin{array}{l}
n \\
k
\end{array}\right]_{p, q} \mathcal{H}_{n-k, p, q}^{(\alpha)}(x, 0 ; u ; \lambda) p^{\left(\begin{array}{l}
k \\
2
\end{array}\right)} \sum_{s=0}^{k}\left(\begin{array}{l}
k \\
s
\end{array}\right) y^{s} a^{k-s} .
\end{aligned}
$$

Proof. Using Definition

$$
\begin{aligned}
& \sum_{n=0}^{\infty} \mathcal{H}_{n, p, q}^{(\alpha)}(x+a, y ; u ; \lambda) \frac{t^{n}}{[n]_{p, q} !} \\
= & \left(\frac{1-u}{\lambda e_{p, q}(t)-u}\right)^{\alpha} E_{p, q}(t y) e_{p, q}((x+a) t) \\
= & \left(\sum_{l=0}^{\infty} \mathcal{H}_{l, p, q}^{(\alpha)}(0, y ; u ; \lambda) \frac{l^{n}}{[l]_{p, q} !}\right) \\
& \times\left(\sum_{k=0}^{\infty} p^{\left(\begin{array}{l}
k \\
2
\end{array}\right)}(x+a)^{k} \frac{t^{k}}{[k]_{p, q} !}\right) \\
= & \left.\sum_{n=0}^{\infty}\left\{\begin{array}{l}
\sum_{k=0}^{n}\left(\left[\begin{array}{l}
n \\
k
\end{array}\right]_{p, q} \mathcal{H}_{n-k, p, q}^{(\alpha)}(0, y ; u ; \lambda)\right) \\
\times p^{k}\left(\begin{array}{l}
k \\
2
\end{array}\right)(x+a)^{k}
\end{array}\right)\right\} \frac{t^{n}}{[n]_{p, q} !} \\
= & \sum_{n=0}^{\infty}\left\{\begin{array}{l}
\sum_{k=0}^{n}\left[\begin{array}{l}
n \\
k
\end{array}\right]_{p, q} \mathcal{H}_{n-k, p, q}^{(\alpha)}(0, y ; u ; \lambda) p^{\left(\begin{array}{l}
k \\
2
\end{array}\right)} \times \sum_{s=0}^{k}\left(\begin{array}{l}
k \\
s
\end{array}\right) y^{s} a^{k-s} \\
t^{n}
\end{array} .\right.
\end{aligned}
$$

Comparing the coefficients of $\frac{t^{n}}{[n]_{p, q} !}$, we have (2.4).

Similarly the other equation is been calculation.

Theorem 2. There is the following relation for the generalized Apostol type q-Frobenius-Euler polynomials

$$
\begin{aligned}
& (2 u-1) \sum_{k=0}^{n}\left[\begin{array}{l}
n \\
k
\end{array}\right]_{p, q} \mathcal{H}_{k, p, q}(0,0 ; u ; \lambda) \mathcal{H}_{n-k, p, q}(x, y ; 1-u ; \lambda) \\
& =u \mathcal{H}_{n, p, q}(x, y ; u ; \lambda)-\mathcal{H}_{n, p, q}(x, y ; 1-u ; \lambda) .
\end{aligned}
$$


Proof. By using the identity

$$
\begin{gathered}
\frac{2 u-1}{\left(\lambda e_{p, q}(t)-u\right)\left(\lambda e_{p, q}(t)-(1-u)\right)} \\
=\frac{1}{\lambda e_{p, q}(t)-u}-\frac{1}{\lambda e_{p, q}(t)-(1-u)}, \\
(2 u-1) \frac{(1-u) e_{p, q}(x t)(1-(1-u)) E_{p, q}(t y)}{\left(\lambda e_{p, q}(t)-u\right)\left(\lambda e_{p, q}(t)-(1-u)\right)} \\
=\frac{(1-u) e_{p, q}(x t) u E_{p, q}(t y)}{\lambda e_{p, q}(t)-u} \\
-\frac{(1-u) e_{p, q}(x t)(1-(1-u)) E_{p, q}(t y)}{\lambda e_{p, q}(t)-(1-u)}, \\
(2 u-1) \sum_{n=0}^{\infty} \mathcal{H}_{n, p, q}(0,0 ; u ; \lambda) \frac{t^{n}}{[n]_{p, q} !} \\
\times \sum_{n=0}^{\infty} \mathcal{H}_{n, p, q}(0,0 ; 1-u ; \lambda) \frac{t^{n}}{[n]_{p, q} !} \\
=u \sum_{n=0}^{\infty} \mathcal{H}_{n, p, q}(x, y ; u ; \lambda) \frac{t^{n}}{[n]_{p, q} !} \\
-(1-u) \sum_{n=0}^{\infty} \mathcal{H}_{n, p, q}(x, y ; 1-u ; \lambda) \frac{t^{n}}{[n]_{p, q} !}
\end{gathered}
$$

Comparing the coefficient of $\frac{t^{n}}{[n]_{p, q} !}$, we prove (2.5).

Remark 1. For $\lim \mathcal{H}_{n, p, q}(x, y ; u ; \lambda)$. Substituting $q \rightarrow 1^{-}$
$p \rightarrow 1$

$\lambda=1, \quad y=0$ in (2.5). We have Carlitz result ([1], equation 2.19).

Theorem 3. There is the following relation for the generalized Apostol type (p, q)-Frobenius-Euler polynomial

$$
\begin{aligned}
& u \mathcal{H}_{n, p, q}(x, y ; u ; \lambda) \\
& =\lambda \sum_{k=0}^{n}\left[\begin{array}{l}
n \\
k
\end{array}\right]_{p, q} \mathcal{H}_{k, p, q}(x, y ; u ; \lambda)-\lambda(1-u)(x+y)_{p, q}^{n} .
\end{aligned}
$$

Proof. By using the identity $e_{p, q}(t) E_{p, q}(-t)=1$,

$$
\frac{u}{\lambda^{2}\left(e_{p, q}(t)-u\right) e_{p, q}(t)}=\frac{1}{\lambda\left(e_{p, q}(t)-u\right)}-\frac{1}{\lambda e_{p, q}(t)} .
$$

We write as

$$
\begin{aligned}
& \frac{u(1-u) e_{p, q}(t x) E_{p, q}(y t)}{\left(\lambda e_{p, q}(t)-u\right) \lambda e_{p, q}(t)} \\
& =\frac{(1-u) e_{p, q}(t x) E_{p, q}(y t)}{\lambda e_{p, q}(t)-u}-\frac{(1-u) e_{p, q}(t x) E_{p, q}(y t)}{\lambda e_{p, q}(t)},
\end{aligned}
$$

$$
\begin{aligned}
& \frac{u}{\lambda} \sum_{n=0}^{\infty} \mathcal{H}_{n, p, q}(x, y ; u ; \lambda) \frac{t^{n}}{[n]_{p, q} ! e_{p, q}(t)} \\
&= \sum_{n=0}^{\infty} \mathcal{H}_{n, p, q}(x, y ; u ; \lambda) \frac{t^{n}}{[n]_{p, q} !} \\
& \quad-\frac{1-u}{\lambda e_{p, q}(t)} e_{p, q}(t x) E_{p, q}(y t), \\
& \frac{u}{\lambda} \sum_{n=0}^{\infty} \mathcal{H}_{n, p, q}(x, y ; u ; \lambda) \frac{t^{n}}{[n]_{p, q} !} \\
&=\sum_{n=0}^{\infty} \mathcal{H}_{n, p, q}(x, y ; u ; \lambda) \frac{t^{n}}{[n]_{p, q} !} \sum_{n=0}^{\infty} \frac{t^{n}}{[n]_{p, q} !} \\
& \quad-(1-u) \sum_{n=0}^{\infty}(x+y)_{p, q}^{n} \frac{t^{n}}{[n]_{p, q} !}
\end{aligned}
$$

Comparing the coefficients of $\frac{t^{n}}{[n]_{p, q} !}$, we have

$$
\begin{aligned}
& u \mathcal{H}_{n, p, q}(x, y ; u ; \lambda) \\
& =\lambda \sum_{k=0}^{n}\left[\begin{array}{l}
n \\
k
\end{array}\right]_{p, q} \mathcal{H}_{k, q}(x, y ; u ; \lambda)-(1-u)(x+y)_{p, q}^{n}
\end{aligned}
$$

\section{Explicit Relation for the Apostol Type $(p, q)$-Frobenius-Euler Polynomials}

Theorem 4. There is the following relation for the Apostol type $(p, q)$-Frobenius-Euler poly-nomials

$$
\begin{aligned}
& \mathcal{H}_{n, p, q}^{(\alpha)}(x, y ; u ; \lambda) \\
& =\frac{1}{1-u} \sum_{k=0}^{n}\left[\begin{array}{l}
n \\
k
\end{array}\right]_{p, q}\left\{\begin{array}{l}
\mathcal{H}_{n-k, p, q}(1, y ; u ; \lambda) \\
-u \mathcal{H}_{k, p, q}(0, y ; u ; \lambda)
\end{array}\right\} \\
& \times \mathcal{H}_{n-k, p, q}^{(\alpha)}(x, 0 ; u ; \lambda) .
\end{aligned}
$$

Proof. Since (1.7);

$$
\begin{aligned}
& \sum_{n=0}^{\infty} \mathcal{H}_{n, p, q}^{(\alpha)}(x, y ; u ; \lambda) \frac{t^{n}}{[n]_{p, q} !} \\
& =\left(\frac{1-u}{\lambda e_{p, q}(t)-u}\right)^{\alpha} e_{p, q}(t x) E_{p, q}(t y) \\
& =\frac{1-u}{\lambda e_{p, q}(t)-u} E_{p, q}(t y) \frac{\lambda e_{p, q}(t)-u}{1-u} \\
& \times\left(\frac{1-u}{\lambda e_{p, q}(t)-u}\right)^{\alpha} e_{p, q}(t x)
\end{aligned}
$$




$$
\begin{aligned}
& =\frac{1}{1-u}\left\{\frac{1-u}{\lambda e_{p, q}(t)-u} E_{p, q}(t y) \lambda e_{p, q}(t)\right. \\
& \times\left(\frac{1-u}{\lambda e_{p, q}(t)-u}\right)^{\alpha} e_{p, q}(t x) \\
& \left.-u\left(\frac{1-u}{\lambda e_{p, q}(t)-u}\right) E_{p, q}(t y)\left(\frac{1-u}{\lambda e_{p, q}(t)-u}\right)^{\alpha} e_{p, q}(t x)\right\} \\
& =\frac{1}{1-u}\left\{\lambda \sum_{k=0}^{\infty} \mathcal{H}_{k, p, q}(1, y ; u ; \lambda) \frac{t^{k}}{[k]_{p, q} !}\right. \\
& \times \sum_{l=0}^{\infty} \mathcal{H}_{l, p, q}^{(\alpha)}(x, 0 ; u ; \lambda) \frac{t^{l}}{[l]_{p, q} !} \\
& -u \sum_{k=0}^{\infty} \mathcal{H}_{k, p, q}(0, y ; u ; \lambda) \frac{t^{k}}{[k]_{p, q} !} \\
& \left.\times \sum_{l=0}^{\infty} \mathcal{H}_{l, p, q}^{(\alpha)}(x, 0 ; u ; \lambda) \frac{t^{l}}{[l]_{p, q} !}\right\} \text {. }
\end{aligned}
$$

Comparing the coefficients of $\frac{t^{n}}{[n]_{p, q} \text { ! }}$, we have (3.1).

Theorem 5. There is the following relation between Apostol type (p, q)-Frobenius-Euler polynomials and the generalized Apostol ( $p, q)$-Bernoulli polynomials

$$
\begin{aligned}
& \mathcal{H}_{n, p, q}^{(\alpha)}(x, y ; u ; \lambda) \\
& =\frac{1}{[n+1]_{p, q}}\left\{\lambda \sum_{r=0}^{n+1}\left[\begin{array}{c}
n+1 \\
r
\end{array}\right]_{p, q}\right. \\
& \times \sum_{k=0}^{r}\left[\begin{array}{c}
r \\
k
\end{array}\right]_{p, q} \mathcal{B}_{n+1-r, p, q}(x, 0 ; \lambda) \\
& \left.-\sum_{k=0}^{n+1}\left[\begin{array}{c}
n+1 \\
k
\end{array}\right]_{p, q} \mathcal{B}_{n+1-k, p, q}(x, 0 ; \lambda)\right\} \\
& \times \mathcal{H}_{k, p, q}^{(\alpha)}(0, y ; u ; \lambda) .
\end{aligned}
$$

Proof.

$$
\begin{aligned}
& \left(\frac{1-u}{\lambda e_{p, q}(t)-u}\right)^{\alpha} e_{p, q}(t x) E_{p, q}(t y) \\
& =\left(\frac{1-u}{\lambda e_{p, q}(t)-u}\right)^{\alpha} E_{p, q}(t y) \frac{t}{\lambda e_{p, q}(t)-1} \frac{\lambda e_{p, q}(t)-1}{t} e_{p, q}(t x), \\
& =\frac{1}{t}\left\{\lambda \sum_{n=0}^{\infty} \sum_{k=0}^{r}\left[\begin{array}{l}
r \\
k
\end{array}\right]_{p, q}\right. \\
& \times \sum_{r=0}^{n}\left[\begin{array}{l}
n \\
r
\end{array}\right]_{p, q} \mathcal{H}_{k, p, q}^{(\alpha)}(0, y ; u ; \lambda) \mathcal{B}_{n-r, p, q}(x, 0 ; \lambda) \\
& \left.-\sum_{n=0}^{\infty} \sum_{k=0}^{n}\left[\begin{array}{l}
n \\
k
\end{array}\right]_{p, q} \mathcal{H}_{k, p, q}^{(\alpha)}(0, y ; u ; \lambda) \mathcal{B}_{n-k, p, q}(x, 0 ; \lambda)\right\} \frac{t^{n}}{[n]_{p, q} !},
\end{aligned}
$$

$$
\begin{aligned}
=\sum_{n=0}^{\infty} \frac{1}{[n+1]_{p, q}} & \left\{\lambda \sum_{r=0}^{n+1}\left[\begin{array}{c}
n+1 \\
r
\end{array}\right]_{p, q}\right. \\
& \times \sum_{k=0}^{r}\left[\begin{array}{l}
r \\
k
\end{array}\right]_{p, q} \mathcal{B}_{n+1-r, p, q}(x, 0 ; \lambda) \\
& \left.-\sum_{k=0}^{n+1}\left[\begin{array}{c}
n+1 \\
k
\end{array}\right]_{p, q} \mathcal{B}_{n+1-k, p, q}(x, 0 ; \lambda)\right\} \\
\times & \mathcal{H}_{k, p, q}^{(\alpha)}(0, y ; u ; \lambda) \frac{t^{n}}{[n]_{p, q} !} .
\end{aligned}
$$

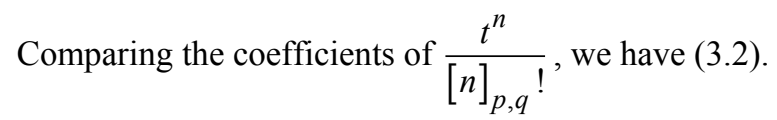

Corollary 1. There is the following relation between Apostol type (p, q)-Frobenius-Euler polynomials and the generalized Apostol ( $p, q)$-Euler polynomials

$$
\begin{aligned}
& \mathcal{H}_{n, p, q}^{(\alpha)}(x, y ; u ; \lambda) \\
& =\frac{1}{2}\left\{\lambda \sum_{k=0}^{r}\left[\begin{array}{l}
r \\
k
\end{array}\right]_{p, q} \sum_{r=0}^{n}\left[\begin{array}{l}
n \\
r
\end{array}\right]_{p, q} \mathcal{E}_{n-r, p, q}(x, 0 ; \lambda)\right. \\
& \left.-\sum_{k=0}^{n}\left[\begin{array}{l}
n \\
r
\end{array}\right]_{p, q} \mathcal{E}_{n-k, p, q}(x, 0 ; \lambda)\right\} \mathcal{H}_{k, p, q}^{(\alpha)}(0, y ; u ; \lambda) .
\end{aligned}
$$

\section{Acknowledgements}

The present investigation was supported, by the Scientific Research Project Administration of Akdeniz University.

\section{References}

[1] Carlitz, L., Eulerian numbers and polynomials, Math. Mag., 32(1959), 247-260.

[2] Carlitz, L., q-Bernoulli numbers and polynomials, Duke Math. J., 15(1948), 987-1050.

[3] Carlitz, L., q-Bernoulli and Eulerian numbers, Trans. Amer. Math. Soc., 76(1954), 332-350.

[4] Cenkci, M., Can, M. and Kurt, V., q-extensions of Genocchi numbers, J. Korean Math. Soc., 43(2006), 183-198.

[5] Cheon, G. S., A note on the Bernoulli and Euler polynomials, Appl. Math. Letter, 16(2003), 365-368.

[6] Duran, U., Acikgoz, M. and Araci, S., On (p, q)-Bernoulli, (p, q)-Euler and (p, q)-Genocchi polynomials, 2016, submitted.

[7] Kim, T., Identities involving Frobenius-Euler polynomials arising from non-linear differential equation, J. Number Theory, 132(2012), 2854-2865.

[8] Kim, T., Some formulae for the q-Bernoulli and Euler polynomials of higher order, J. Math. Analy. Appl., 273(2002), 236-242.

[9] Kurt, B., A Note on the Apostol type q-Frobenius-Euler Polynomials and Generalizations of the Srivastava-Pinter Addition Theorems, Filomat, 2016, 30(1), 65-72.

[10] Kurt, B. and Simsek Y., Frobenius-Euler type polynomials related to Hermite-Bernoulli polynomials, Numerical Analysis and Appl. Math. ICNAAM 2011 Conf. Proc., 1389(2011), 385-388.

[11] Kurt, B. and Simsek Y., On the generalized Apostol type Frobenius-Euler polynomials, Adv. in Diff. Equ.

[12] Kac, V. and Cheung, P., Quantum Calculus, Springer (2002). 
[13] Luo, Q.-M. and Srivastava, H. M., Some relationships between the Apostol-Bernoulli and Apostol-Euler polynomials, Comp. Math. App., 51(2006), 631-642.

[14] Luo, Q.-M., Some results for the q-Bernoulli and q-Euler polynomials, J. Math. Anal. Appl., 363 (2010), 7-18.

[15] Mahmudov, N. I., q-analogues of the Bernoulli and Genocchi polynomials and the Srivastava-Pintér addition theorems, Discrete Dynamics in Nature and Soc., Article number 169348, 2012

[16] Mahmudov, N. I., On a class of q-Bernoulli and q-Euler polynomials, Adv. in Diff. Equa., 2013.

[17] Simsek, Y., Generating functions for q-Apostol type FrobeniusEuler numbers and polynomials, Axioms, 1(2012), 395-403.

[18] Simsek, Y., Generating functions for generalized Stirling type numbers Array type polynomials, Eulerian type polynomials and their applied, Arxiv: 1111.3848v1.2011.

[19] Srivastava, H. M., Some generalization and basic (or q-) extensions of the Bernoulli, Euler and Genocchi polynomials, Appl. Mah. Inform. Sci., 5(2011), 390-444.
[20] Srivastava, H. M., Kurt, B. and Simsek, Y., Some families of Genocchi type polynomials and their interpolation function, Integral Trans. and Special func., 23(2012).

[21] Srivastava, H. M., Garg, M. and Choudhary, S., A new genralization of the Bernoulli and related polynomials, Russian J. Math. Phys., 17(2010), 251-261.

[22] Srivastava H. M., Garg M. and Choudhary S., Some new families of the generalized Euler and Genocchi polynomials, Taiwanese J. Math., 15(2011), 283-305.

[23] Srivastava, H. M. and Choi, J., Series associated with the zeta and related functions, Kluwer Academic Publish, London (2011).

[24] Srivastava, H. M. and Pintér, A., Remarks on some relationships between the Bernoulli and Euler polynomials, Appl. Math. Letter, 17(2004), 375-380.

[25] Trembley, R., Gaboury, S. and Fugére, B. J., A new class of generalized Apostol-Bernoulli polynomials and some analogues of the Srivastava-Pintér addition theorems, Appl. Math. Letter, 24(2011), 1888-1893. 